\title{
Cyclotron radiation cooling of a short electron bunch kicked in an undulator with guiding magnetic field
}

\author{
I. V. Bandurkin, ${ }^{1}$ I. V. Osharin, ${ }^{1}$ and A. V. Savilov ${ }^{1,2}$ \\ ${ }^{1}$ Institute of Applied Physics, Russian Academy of Sciences, Nizhny Novgorod 603950, Russia \\ ${ }^{2}$ Lobachevsky State University of Nizhny Novgorod, Nizhny Novgorod 603950, Russia
}

(Received 30 June 2015; published 20 November 2015)

\begin{abstract}
We propose to use of an undulator with the guiding axial magnetic field as a "kicker" forming a bunch of electron gyro-oscillators with a small spread in the axial velocity. The cyclotron emission from the bunch leads to losing oscillatory velocity of electron gyrorotation, but it does not perturb the axial electron velocity. This effect can be used for transformation of minimization of the spread in electron axial velocity in the undulator section into minimization of the spread in electron energy in the cyclotron radiation section.
\end{abstract}

DOI: 10.1103/PhysRevSTAB.18.110702

PACS numbers: 41.60.Cr, 41.60.Ap

\section{INTRODUCTION}

Fast development of the technique of photocathode electron photoinjectors has resulted in creation of compact and accessible sources of moderately relativistic (several $\mathrm{MeV}$ ) dense (up to $1 \mathrm{nC}$ in a ps pulse) electron bunches [1-3]. Methods providing a decrease in the energy spread (cooling) are actual from the point of view of various applications of such electron bunches, including freeelectron lasers (FELs) [4-6]. However, cooling methods are developed now basically for electron beams of very high energies [7-12]. As for moderately relativistic (several or tens $\mathrm{MeV}$ ) high-dense short electron bunches, the strong Coulomb interaction of the particles results in a requirement for a short length of a cooling system. Therefore, in this situation, the cooling system should possess resonant properties, namely, a strong dependence of parameters of the particles inside the cooling system on their input energies.

An example of such a resonant system is a periodic magnetistatic undulator immersed in a uniform guiding axial magnetic field [13]. Such undulator systems are used in mm-wavelength FELs $[4,14]$ to decrease sensitivity of these devices to the velocity spread. An important peculiarity of such a system is a resonant character of the dependence of the undulator velocity on the electron energy. This dependence has the "normal" character (namely, the decreasing one) at relatively low values of the guiding magnetic field, when the electron cyclotron frequency corresponding to this field is lower than the bounce frequency of electron oscillations in the periodic undulator field. In contrast, at high axial magnetic fields

Published by the American Physical Society under the terms of the Creative Commons Attribution 3.0 License. Further distribution of this work must maintain attribution to the author $(s)$ and the published article's title, journal citation, and DOI. this dependence has the "abnormal," increasing character, so that particles with higher input energies obtain bigger undulator velocities.

It is shown in [13] that the abnormal dependence of the undulator velocity on the electron energy can be used to provide "axial cooling" of the electron bunch; this term means minimization of the spread in axial electron velocity inside the undulator due to transformation of the input spread in axial velocity into the spread in undulator velocity. It is mentioned also in [13] that the axial cooling can be, in principle, transformed into "real" cooling of the electron bunch (minimization of the spread in total electron energy) due to cyclotron emission from gyrorotating electrons, which obtain their oscillatory velocities in the axial cooling undulator (so that the undulator is used as a "kicker" imparting electrons their rotatory velocities). In this paper, we study this idea in detail. In Sec. II, we describe the process of axial cooling of a moderately relativistic electron bunch in a magnetostatic undulator with guiding axial magnetic field, as well as discuss the use of an rf-wave undulator in the case of higher electron energies. Cyclotron emission from a short electron bunch, which is kicked in the axial cooling undulator, as well as motion of electrons in the radiated field is studied in Sec. III.

\section{AXIAL COOLING UNDULATOR}

A system composed by a periodic magnetic field (undulator) and a uniform magnetic field can be used to provide axial cooling of an electron bunch, namely, a significant decrease in the spread in axial electron velocity by means of "transforming" this spread into the spread in transverse velocity [13]. This is possible, if the dependence of the velocity of undulator electron oscillations on the axial electron velocity, $V_{u}\left(V_{\|}\right)$, has the abnormal character, $\partial V_{u} / \partial V_{\|}>0$. 
(a)

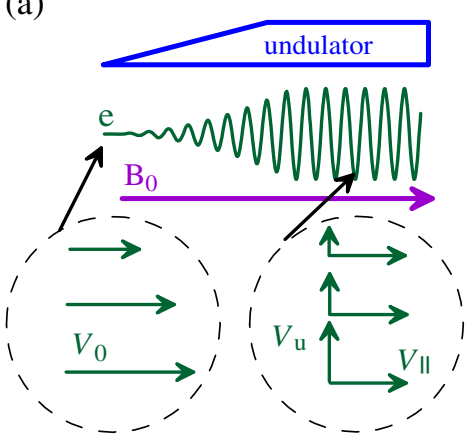

(b)

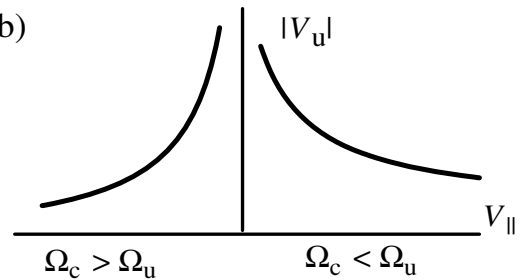

FIG. 1. (a) Minimization of the spread in axial velocity in a circular-polarized magnetostatic undulator with guiding magnetic field. (b) Resonant dependence of the undulator velocity on the axial electron velocity.

Let us consider a scheme shown in Fig. 1(a). Electrons move along the axial magnetic field and enter into a circularly polarized undulator, where they get some rotatory velocity. In order to avoid appearance of "parasitic" cyclotron oscillations, the undulator includes an input section, where its field adiabatically slowly grows with the coordinate. If any electron at the input of the system possesses only axial velocity, $V_{0}=\bar{V}_{0}+\delta V_{0}$, then axial velocity in the regular undulator region is determined by the energy conservation low: $V_{\|}^{2} \approx V_{0}^{2}-V_{u}^{2}$, where $V_{u}$ is the velocity of electron rotation in the undulator. Thus,

$$
\begin{aligned}
V_{\|}^{2} & \approx \bar{V}_{0}^{2}+2 \bar{V}_{0} \times \delta V_{0}-V_{u}^{2}\left(\bar{V}_{0}\right)-\alpha \times \delta V_{0}, \\
\alpha & =\partial V_{u}^{2} / \partial V_{\|} .
\end{aligned}
$$

The condition of the axial cooling, $\alpha=2 \bar{V}$, corresponds to the minimal spread in $V_{\|}$. This condition is independent on the initial spread, $\delta V_{0}$.

Evidently, in order to provide axial cooling at moderate undulator fields, one should use the close-to-resonance range of parameters, where $\partial V_{u} / \partial V_{\|}$is great enough [Fig. 1(b)]. In addition, $\partial V_{u} / \partial V_{\|}$should be positive, so that initial axial velocity excess, $\delta V_{0}$, should be compensated by greater rotatory velocity, $V_{u}$. This is possible due to the presence of the axial magnetic field, $\mathbf{B}_{0}=\mathbf{z} B_{0}$. In this case, a particle represents a free cyclotron oscillator (with the frequency $\Omega_{c}=e B_{0} / m c \gamma$ ), which is under acting of the periodic undulator force (with the bounce frequency $\Omega_{u}=h_{u} V_{\|}$, where $h_{u}$ is the undulator wavelength). Due to this fact, the dependence $V_{u}\left(V_{\|}\right)$has a resonant character
[Fig. 1(b)], namely, is a decreasing function at $\Omega_{u}>\Omega_{c}$ and an increasing function at $\Omega_{u}<\Omega_{c}$ [4,14]. We should operate in the region $\Omega_{u}<\Omega_{c}$, where electrons with higher input energies get bigger oscillatory velocities $\left(\partial V_{u} / \partial V_{\|}>0\right)$; this is similar to the "negative-mass" effect well known in theory of cyclotron accelerators $[15,16]$ and cyclotron resonance masers [17-24].

We consider electron motion along a helical trajectory in the axial uniform magnetic field, $\mathbf{B}_{0}=\mathbf{z} B_{0}$, and in the quasiperiodic transverse field of a circularly polarized undulator, $B_{x}+i B_{y}=B_{u}(z) \exp \left(i h_{u} z\right)$. The motion equation for the complex transverse momentum of a particle, $p_{+}=p_{x}+i p_{y}=\gamma \beta_{+}$, has the following form:

$$
\frac{d p_{+}}{d z}=i h_{c} \frac{p_{+}}{p_{\|}}+i h_{u} K(z) \exp \left(i h_{u} z\right) .
$$

Here, $\mathbf{p}=\gamma \boldsymbol{\beta}, \boldsymbol{\beta}=\mathbf{V} / c, \gamma=\left(1-\boldsymbol{\beta}^{2}\right)^{-1 / 2}$ is the relativistic electron Lorentz factor, $h_{c}=e B_{0} / m c^{2}=\gamma \Omega_{c} / c$ is the normalized axial magnetic field, and $K(z)=-e B_{u} /$ $h_{u} m c^{2}$ is the normalized undulator field (the so-called undulator factor at the zero axial field $B_{0}=0$ ); its dependence on the axial coordinate describes a smooth enter of particles into the undulator. In the simplest model, electrons have only axial velocities at the beginning of the system, $\boldsymbol{\beta}_{0}=\mathbf{z} \beta$, with some spread, $\bar{\beta}_{0}-\delta \beta_{0}<\beta_{0}<\bar{\beta}_{0}+\delta \beta_{0}$.

If the entry into the undulator is smooth enough, then the growth of the forced undulator oscillations does not induce the free cyclotron oscillations of particles. Therefore, in the regular region $K=$ const the transverse electron velocity is described as follows:

$$
\beta_{+}=p_{+} / \gamma_{0}=\beta_{u} \exp \left(i h_{u} z\right)
$$

where

$$
\beta_{u}=K / \gamma_{0} \Delta
$$

is the velocity of electron rotation in the undulator field, and $\Delta=1-\Omega_{c} / \Omega_{u}$ is the mismatch between the electron cyclotron frequency and the bounce frequency of the undulator oscillations. The initial axial velocity is related with the axial velocity in the regular undulator by the energy conservation law:

$$
\beta_{0}^{2}=\beta_{\|}^{2}+\beta_{u}^{2}
$$

Evidently,

$$
\beta_{\|} \approx f\left(\bar{\beta}_{0}\right)+\left(\beta_{0}-\bar{\beta}_{0}\right) f^{\prime}
$$

where function $\beta_{\|}=f\left(\beta_{0}\right)$ is determined by Eqs. (4) and (2). Having differentiated Eq. (4), one obtains 


$$
\beta_{0}=\beta_{\|} f^{\prime}-\frac{K^{2}}{\left(\gamma_{0}-h_{c} / \beta_{\|} h_{u}\right)^{3}}\left(\gamma_{0}^{3} \beta_{0}+\frac{h_{c}}{h_{u}} \frac{f^{\prime}}{\beta_{\|}^{2}}\right) .
$$

According to Eq. (5), the spread in $\beta_{\|}$is minimal, when $f^{\prime}=\partial \beta_{\|} / \partial \beta_{0}=0$. In the ultrarelativistic limit, $\gamma \gg 1$, this leads to the following condition [13]:

$$
K^{2} \approx-\Delta^{3} \text {. }
$$

In this paper, the axial cooling undulator is considered as a specific kicking system, which imparts to electrons the velocity of free cyclotron rotation in the axial homogeneous magnetic field. According to Eq. (1), if the undulator section possesses a sharp exit, then the exit of electrons from the undulator leads to transformation of the forced oscillations in the undulator field, $\beta_{+}=\beta_{u} \exp \left(i h_{u} z\right)$, into free cyclotron oscillations,

$$
\beta_{+}=\beta_{\perp} \exp \left(i \int \Omega_{\mathrm{c}} d t\right)
$$

with the same oscillatory velocity $\beta_{\perp}=\beta_{u}$ (Fig. 2). Thus, at the beginning of the radiation section the electron bunch has some spread in cyclotron rotation velocity, but the spread in axial velocity is minimal. The particles lose their transverse velocities due to the radiation, such that in the optimal situation electrons possess only axial velocity at the end. It is important that axial velocities stay constant during this process (see Sec. III). Thus, if the spread in axial velocity is minimized in the beginning of the radiation section [Fig. 1(a)], then the radiation process results in minimization of the spread in the total velocity (Fig. 2).

According to Eq. (7), in order to provide axial cooling at moderate undulator fields, $K<1$, one should use the closeto-resonance range of parameters, $|\Delta|<1$. In the case of a magnetostatic undulator, the axial magnetic field should be so high that the corresponding electron cyclotron frequency

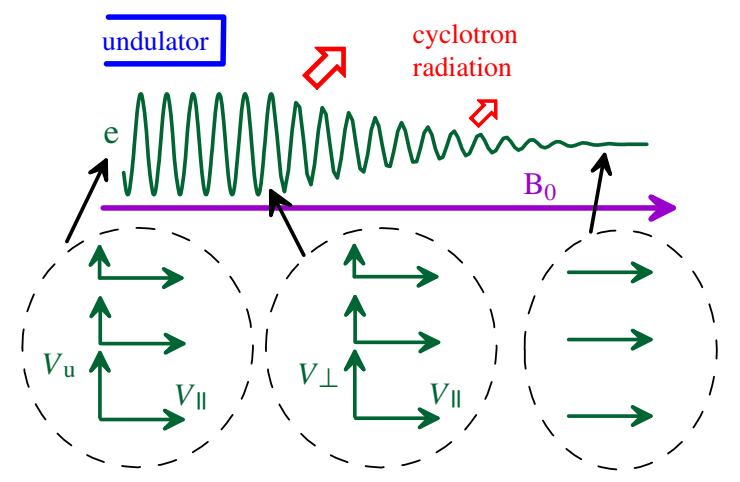

FIG. 2. Transformation of the axial cooling (minimization of the spread in axial electron velocity) in the undulator section into the real cooling (minimization of the spread in electron energy) in the radiation section. should be of the order of (and slightly greater than) the undulator bounce frequency,

$$
\Omega_{c} \sim \Omega_{u} .
$$

This formula leads to the following estimation:

$$
B_{0} \sim \frac{\gamma}{\lambda_{u}}
$$

where the axial magnetic field $B_{0}$ and the undulator period $\lambda_{u}$ are measured in Tesla and $\mathrm{cm}$, respectively. At relatively low electron energies $(\gamma \sim 10)$, the undulator period $\lambda_{u}=$ $5 \mathrm{~cm}$ corresponds to a moderate (especially for pulsed systems) axial magnetic field of $B_{0} \approx 2 \mathrm{~T}$.

A possible way to decrease the magnetic field required at higher electron energies is the use a powerful rf pulse propagating together with electrons instead of a magnetostatic undulator. In the case of the rf undulator, the bounce frequency is determined as follows:

$$
\Omega_{u}=\omega_{u}-h_{u} V_{\|}=\omega_{u}\left(1-\beta_{\mathrm{gr}, u} \beta_{\|}\right),
$$

where $\beta_{\mathrm{gr}, u}=V_{\mathrm{gr}, u} / c=c h_{u} / \omega_{u}$ is the normalized group velocity of the undulator wave. In this situation, the Doppler conversion factor leads to a significant decrease in the value of the required magnetic field:

$$
B_{0} \sim \frac{\gamma}{\lambda_{u}}\left(1-\beta_{\mathrm{gr}, u} \beta_{\|}\right),
$$

where $\lambda_{u}$ is the wavelength measured in $\mathrm{cm}$.

\section{CYCLOTRON RADIATION SECTION}

We consider the cyclotron rf emission from a short electron bunch moving in a circular cross-section waveguide [Fig. 3(a)]. The electron move along helical trajectories is due to gyrorotations of particles in the guiding magnetic field $\mathbf{B}=\mathbf{z} B_{0}$ around the waveguide axis. In order to provide coherent character of the spontaneous radiation, the axial bunch length, $L_{e}$, should be shorter than the characteristic wavelength of the radiated wave, $L_{e}<\lambda$. In this situation, the bunch radiates a wave packet with an axial length, which is significantly longer that the bunch length [Fig. 3(b)]. It will be shown further that the optimal (from the points of view of both the rate and the quality of the cooling process) situation is realized, when the radiated wave packet propagates together with the electron bunch, such that the axial electron velocity, $V_{\|}$, should be close to the group velocity of the rf wave, $V_{\mathrm{gr}}$.

Let us introduce a polar system of coordinates, $x+i y=r e^{i \phi}$. Since every electron rotates around the waveguide axis, its polar phases coincides with the gyrorotation phase, and the complex oscillatory velocity is determined as follows: 

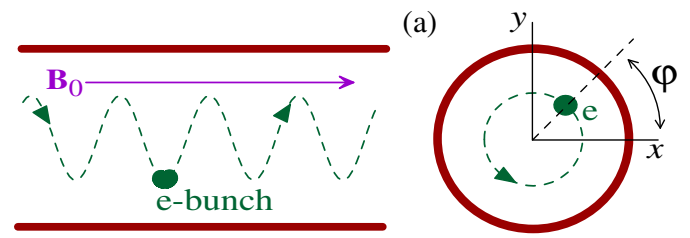

(b)

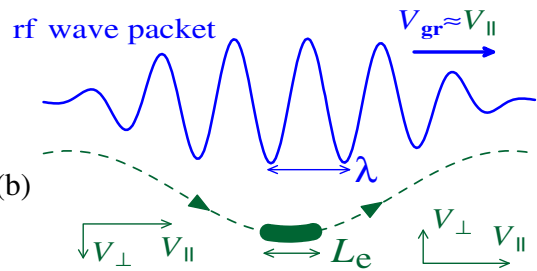

FIG. 3. (a) Motion of the electron bunch of gyrorotating electrons inside the waveguide of the radiation section, and (b) a short electron bunch on the background of the radiated rf-wave packet in the case of the group synchronism.

$$
V_{+}=i V_{\perp} \exp (i \phi), \quad \phi \approx \phi_{0}+\int_{0}^{t} \Omega_{c} d t
$$

The interaction between an axis-encircling electron beam and a mode of a circular waveguide at the fundamental cyclotron resonance is possible, if the azimuthal index of the mode is equal to $1[25,26]$. We consider a wave, which has a fixed transverse structure corresponding to the lowest possible $\mathrm{TE}_{1,1}$ transverse mode. The electric and magnetic components of the field of this mode

$$
\mathrm{E}=\frac{-1}{c} \frac{\partial \mathbf{A}}{\partial t}, \quad \mathrm{~B}=\operatorname{rot} \mathbf{A}
$$

are described by the following vector potential:

$$
\mathbf{A}=\operatorname{Re} A(z, t) \mathbf{F}(r, \phi) \exp \left(i \omega_{0} t-i h_{0} z\right)
$$

where

$$
\mathbf{F}=\frac{-i}{k_{0} k_{\perp}} \operatorname{rot}\left[\mathbf{z}_{0} J_{1}\left(k_{\perp} r\right) \exp (-i \phi)\right]
$$

is the membrane function corresponding to the mode $\mathrm{TE}_{1,1}$. In these formulas, $\omega_{0}$ is a basic frequency, $k_{0}=\omega_{0} / c$, and $h_{0}=\sqrt{k_{0}^{2}-k_{\perp}^{2}}$ is the axial wave number corresponding to the basic frequency, which is chosen so that it satisfies exactly the cyclotron resonance condition,

$$
\omega_{0}=h_{0} V_{\|}+\Omega_{c 0},
$$

where $\Omega_{c 0}=e B_{0} / m c \gamma_{0}$ is the initial cyclotron frequency. It is important that the emission from a short electron bunch possesses a nonmonochromatic spatiotemporal character. We suppose that the frequency spectrum of the radiated $\mathrm{rf}$ signal is narrow, $\delta \omega \ll \omega_{0}$. These two statements are described by the assumption that the complex rf wave amplitude in Eq. (12), $A(z, t)$, is a slow function of both the axial coordinate and the time.

\section{A. Equations of electron motion}

Let us notice that Eq. (12) describes the rf field, which is similar to a plane circularly polarized wave in the center of the waveguide (in the point of injection of the electron bunch):

$\left.E_{+}\right|_{r \rightarrow 0} \approx \frac{i}{2} A(z, t) \exp \left(i \omega_{0} t-i h_{0} z\right), \quad B_{+} \approx i \frac{h_{0}}{k_{0}} E_{+}$.

In this case, the equation for the relativistic electron energy,

$$
m c^{2} \frac{d \gamma}{d z}=-\frac{e}{V_{\|}} \operatorname{Re} E_{+} V_{+}^{*}
$$

leads to the following normalized equation:

$$
\frac{1}{k_{0}} \frac{d \gamma}{d z}=-\frac{p_{\perp}}{2 p_{\|}} \operatorname{Re} a(z, t) \exp (i \varphi),
$$

where $a=e A / m c^{2} k_{0}$ is the dimensionless slow wave amplitude, and $\varphi=\omega_{0} t-h_{0} z-\phi$ is the wave phase on the basic frequency.

The equation for the axial electron momentum has the following form:

$$
m c \frac{d p_{\|}}{d z}=-\frac{e}{c V_{\|}} \operatorname{Im} B_{+} V_{+}^{*} .
$$

This equation together with Eqs. (14) and (15) leads to the following simple relations between changes in the energy and in the axial momentum [15,16,27-31]:

$$
\frac{d p_{\|}}{d z}=\beta_{\mathrm{gr}} \frac{d \gamma}{d z},
$$

where $\beta_{\mathrm{gr}}=V_{\mathrm{gr}} / c=h_{0} / k_{0}$ is the group velocity of the radiated wave normalized to the speed of light. This fact has an evident quantum interpretation, namely, the elementary act of radiation of the wave by a particle is emission of a quant with energy $\hbar \omega$ and axial momentum $\hbar h$, so that $\delta p_{\|} / \delta \gamma=\beta_{\mathrm{gr}}$.

Equation (18) leads to the following equation describing the change in the axial electron velocity:

$$
\frac{d \beta_{\|}}{d z}=\frac{d}{d z}\left(\frac{p_{\|}}{\gamma}\right)=\frac{d \gamma}{d z} \times \frac{\beta_{\mathrm{gr}}-\beta_{\|}}{\gamma} .
$$

If the group velocity coincides with the axial electron velocity, $\beta_{\mathrm{gr}}=\beta_{\|}$, then the axial electron velocity is 
constant, and the cyclotron radiation leads to the loss in the transverse component of the electron velocity only. Let us suppose that at the end of the radiation section the particles lose all their initial oscillatory velocity. If the axial cooling (minimization of the spread in $\beta_{\|}$) is provided at the input of the radiation section, then the radiation process transforms this axial cooling into the real cooling (minimization of the spread in the total energy) (Fig. 2).

The equation for the change in the normalized transverse momentum of a particle follows from the general relativistic relation $\gamma^{2}=1+p_{\perp}^{2}+p_{\|}^{2}$ and Eq. (20):

$$
\frac{d p_{\perp}}{d z}=\frac{1}{p_{\perp}} \frac{d}{d z}\left(\gamma^{2}-p_{\|}^{2}\right)=\frac{d \gamma}{d z} \times \frac{\gamma\left(1-\beta_{\mathrm{gr}} \beta_{\|}\right)}{p_{\perp}} .
$$

Having taken into account Eq. (16), one obtains

$$
\frac{1}{k_{0}} \frac{d p_{\perp}}{d z}=-\frac{1-\beta_{\mathrm{gr}} \beta_{\|}}{2 \beta_{\|}} \times \operatorname{Re} a(z, t) \exp (i \varphi) .
$$

Since $\beta_{\mathrm{gr}}=\beta_{\|}$and $\beta_{\|}=$const, in the ultrarelativistic approximation one obtains

$$
\frac{1}{k_{0}} \frac{d p_{\perp}}{d z} \approx \frac{-1}{2 \gamma_{0}^{2}} \times \operatorname{Re} a(z, t) \exp (i \varphi) .
$$

In the right-hand part of the following equation for the electron phase with respect to the radiated wave,

$$
\frac{1}{k_{0}} \frac{d \varphi}{d z}=\frac{1}{k_{0}} \frac{d}{d z}\left(\omega_{0} t-h_{0} z-\phi\right)=\frac{1}{\beta_{\|}}-\beta_{\mathrm{gr}}-\frac{\Omega_{c 0}}{\beta_{\|} \omega_{0}} \times \frac{\gamma_{0}}{\gamma},
$$

only the last term varies in the process of the rf-wave radiation. Having taken into account both cyclotron resonance condition (13) and group synchronism condition $\beta_{\mathrm{gr}}=\beta_{\|}$, one obtains

$\frac{1}{k_{0}} \frac{d \varphi}{d z}=-\frac{\Omega_{c 0}}{\beta_{\|} \omega_{0}} \times\left(\frac{\partial}{\partial \gamma} \frac{\gamma_{0}}{\gamma}\right) \times\left(\gamma-\gamma_{0}\right)+\Delta \approx \frac{\gamma-\gamma_{0}}{\gamma_{0}^{3}}+\Delta$.

Let us assume that at the beginning of the radiation section, $z=0$, electrons have the same phases of their cyclotron rotation, $\phi$. Then the initial phase of a particle, $\varphi(z=0)=\varphi_{0}=\omega_{0} t_{0}$, is determined by the time of its entry into the radiation section. Thus, the phase size of the electron bunch is determined by the ratio between its length and the characteristic wavelength of the radiated wave:

$$
0 \leq \varphi_{0} \leq \delta \varphi_{0}, \quad \delta \varphi_{0}=2 \pi L_{e} / \lambda .
$$

In Eq. (22), the mismatch of the cyclotron resonance, $\Delta=\frac{1}{\beta_{\|}}-\beta_{\mathrm{gr}}-\frac{\Omega_{c 0}}{\beta_{\|} \omega_{0}}$, describes the possible effect of the spread in electron velocity of the process of the electron-wave interaction. If the spread is absent, then one should put $\Delta=0$ in Eq. (22). It is seen from the formula for $\delta=0$ (and well known from the cyclotron-resonance maser theory [29-31]) that the most important effect making worse the cyclotron interaction of relativistic electrons with a traveling wave is the spread in axial electron velocity, $\beta_{\|}$. Thus, the use of the axial cooling undulator as a kicker imparting to electrons their cyclotron oscillations is a way to solve the problem of sensitivity of the cyclotron maser to the spread in axial electron velocity. Therefore, the cyclotron maser with the axial cooling kicker proposed in this paper can be attractive not as a way to provide a cooling cyclotron radiator, but also from the more general point of view, namely, the realization of a relativistic electron cyclotron source of a short-wavelength radiation as itself.

One should notice that equations of electron motion used in our model do not include the rf space-charge effects. An analytical description of these effects for a moving bunch of a complicated form (a piece of helix) is difficult, but there are two reasons to neglect these effects (at least in the simplest model). Both of them are due to the fact that in the mechanism of electron bunching in the cyclotron masers significantly differ from this mechanism taking place in the electron maser based with the axial electron bunching, namely, free-electron masers and Cherenkov (Smith-Purcell) devices. In cyclotron masers, the electron bunching the $2 \mathrm{D}$ character, namely, this is a combination of the axial bunching (caused by the dependence of the axial electron velocity on the energy) and the azimuthal bunching (caused by the dependence of the electron cyclotron velocity on the energy). It is important that axial and azimuthal bunching mechanisms have "opposite signs," so that there is a partial compensation of these two kinds of bunching; this compensation is described by the difference $\left(1-\beta_{\mathrm{gr}} \beta_{\|}\right) \approx \gamma_{0}^{-2}$ in Eq. (22).

Thus, the first reason to neglect the rf space-charge effects is their weakness. Actually, due to the partial compensation of the two different kinds of bunching, a "compensation" factor $\left(1-\beta_{\mathrm{gr}} \beta_{\|}\right) \approx \gamma_{0}^{-2}$ appears in the space-charge parameter in the theory of the cyclotron resonance maser [24]. Moreover, in the case of the cyclotron excitation of a wave with the group velocity comparable with the speed of light, there is one more effect weakening the rf space-charge forces. This is a complicated (helical) spatial structure of the electron bunches [18-24] caused by rotating character of the excited wave. According to the theory [24], this effect is described also by a factor of $\approx \gamma_{0}^{-2}$. Finally, as compared to the cases of both free-electron and Cherenkov masers, the space-charge parameter in the case of the cyclotron maser is smaller by a factor of $\gamma_{0}^{-4}$. The second reason to neglect the rf space-charge effects is the "negative-mass" character of these effects [18-24]. Since the azimuthal bunching is stronger than the axial one, the quasi-Coulomb interaction of electrons leads not to their mutual repulsion (as it happens free-electron and Cherenkov masers) but to their effective phase attraction. 


\section{B. rf wave emission}

In order to describe the spatiotemporal evolution of the slow rf wave amplitude $a(z, t)$, we consider the wave equation,

$$
\frac{1}{c^{2}} \frac{\partial^{2} \mathbf{A}}{\partial t^{2}}-\frac{\partial^{2} \mathbf{A}}{\partial z^{2}}-\Delta_{\perp} \mathbf{A}=\frac{4 \pi}{c} \mathbf{j},
$$

with the electron current density,

$$
j_{+}=j_{x}+i j_{y}=\rho\left\langle V_{+}\right\rangle \times f\left(z-V_{\|} t\right) \times \delta(r) .
$$

Here, $\rho$ is the electron density, $\left\langle V_{+}\right\rangle$is the averaged complex transverse velocity, $\langle\cdots\rangle$ denotes averaging over all electrons having the same z-coordinate, and $f\left(z-V_{\|} t\right)$ describes the position of the electron bunch: $f=1$ at $0 \leq z-V_{\|} t \leq L_{e}$, otherwise $f=0$. The wave equation leads to the following equation for the slow rf wave amplitude:

$$
\begin{aligned}
& \frac{1}{c^{2}} \frac{\partial^{2} a}{\partial t^{2}}+\frac{2 i \omega_{0}}{c^{2}} \frac{\partial a}{\partial t}+2 i h_{0} \frac{\partial a}{\partial z}-\frac{\partial^{2} a}{\partial z^{2}} \\
& \quad=i k_{0}^{2} \frac{2 I}{N I_{A}}\left(1-\beta_{\mathrm{gr}}^{2}\right) \times \hat{j} \times f\left(z-V_{\|} t\right) .
\end{aligned}
$$

Here, $N \approx 0.404$ is the norm of the $\mathrm{TE}_{1,1}$ transverse mode, and $\hat{j}(z, t)=\left\langle\beta_{\perp} \exp (-i \varphi)\right\rangle$ is the electron-current harmonic.

Similar to works [32,33] devoted to the cyclotron superradiation of a short electron bunch, we introduce the following normalized variables:

$$
\zeta=k_{0} \times\left(z-V_{\|} t\right), \quad \tau=\omega_{0} \times\left(t-V_{\|} z / c^{2}\right) .
$$

Then, Eq. (24) is transformed as follows:

$\frac{\partial^{2} a}{\partial \tau^{2}}-\frac{\partial^{2} a}{\partial \zeta^{2}}+2 i s \frac{\partial a}{\partial \tau}-2 i \varepsilon \frac{\partial a}{\partial \zeta}=i g \frac{2 I}{N I_{A}} \times \hat{j} \times f(\zeta)$.

Here, $g=\left(1-\beta_{\mathrm{gr}}^{2}\right) /\left(1-\beta_{\|}^{2}\right), s=\left(1-\beta_{\mathrm{gr}} \beta_{\|}\right) /\left(1-\beta_{\|}^{2}\right)$, and $\varepsilon=\left(\beta_{\|}-\beta_{\mathrm{gr}}\right) /\left(1-\beta_{\|}^{2}\right)$ are factors determined by the axial electron velocity and the group velocity of the radiated wave.

Further, we consider the situation, when the group synchronism condition is fulfilled at least approximately, $\beta_{\mathrm{gr}} \approx \beta_{\|}$. In this case, $g \approx s \approx 1$, whereas the factor describing the slippage of the wave with respect to electrons,

$$
\varepsilon \approx \gamma_{0}^{2}\left(\beta_{\|}-\beta_{\mathrm{gr}}\right),
$$

can be small. In addition, we consider emission from a very short electron bunch, $L_{e} \ll \lambda$. In this case, function $f(\zeta)$ can be approximated as follows [see also Eq. (23)]:

$$
f(\zeta) \approx k_{0} L_{e} \delta(\zeta) \approx \delta \varphi_{0} \delta(\zeta),
$$

and wave equation (26) is reduced as follows [32,33]:

$$
2 i \frac{\partial a}{\partial \tau}+2 i \varepsilon \frac{\partial a}{\partial \zeta}-\frac{\partial^{2} a}{\partial \zeta^{2}}=i G \times \hat{j}(\tau) \times \delta(\zeta),
$$

with $G=\frac{2 I}{N I_{A}} \delta \varphi_{0}$. Let us notice that in this delta-function approximation, the electron current harmonic is determined by the same formula,

$$
\hat{j}(\tau)=\left\langle\beta_{\perp} \exp (-i \varphi)\right\rangle,
$$

but $\langle\cdots\rangle$ denotes averaging over all electrons of the bunch. Thus, in order to provide the start of the spontaneous rfwave emission, the initial phase size of the electron bunch should be small enough: $\delta \varphi_{0}<2 \pi$ and, therefore, $L_{e}<\lambda$.

In the left-hand part of Eq. (28), $\frac{\partial^{2} a}{\partial \tau^{2}}$ has been omitted, as this is small as compared to $\frac{\partial a}{\partial \tau}$. As for the term $\frac{\partial^{2} a}{\partial \zeta^{2}}$ cannot be omitted if the slippage factor $\varepsilon$ is small. Otherwise, in the approximation of a large slippage factor $\left(\varepsilon \frac{\partial a}{\partial \zeta} \gg \frac{\partial^{2} a}{\partial \zeta^{2}}\right)$, one easily obtains the following solution of Eq. (28):

$$
a \approx \frac{G}{2 \varepsilon} \times \hat{j}(\tau-\zeta / \varepsilon),
$$

which describes quasistationary spontaneous emission from the electron bunch.

In the general case, the solution of Eq. (28) cam be obtained after the following substitution:

$$
a(\zeta, \tau)=a_{1}(\zeta, \tau) \exp \left(-i \varepsilon^{2} \tau / 2-i \varepsilon \zeta\right) .
$$

This transforms Eq. (27) into the Schrödinger equation,

$$
2 i \frac{\partial a_{1}}{\partial \tau}-\frac{\partial^{2} a_{1}}{\partial \zeta^{2}}=S(\zeta, \tau),
$$

with the following source:

$$
S(\zeta, \tau)=i G \times \hat{j}(\tau) \times \exp \left(i \varepsilon^{2} \tau / 2+i \varepsilon \zeta\right) \times \delta(\zeta) .
$$

At the zero initial condition, $a_{1}(\tau=0, \zeta)=0$, the solution of Eq. (31) can be found analytically [34],

$$
\begin{aligned}
a_{1}(\zeta, \tau)= & \frac{1}{2 \sqrt{i \pi}} \int_{0}^{t} \frac{1}{\sqrt{\tau-\tau^{\prime}}}\left\{\int_{-\infty}^{+\infty} S\left(\zeta^{\prime}, \tau^{\prime}\right)\right. \\
& \left.\times \exp \left[-\sqrt{2 i}\left(\zeta^{\prime}-\zeta\right)^{2} / 4\left(\tau-\tau^{\prime}\right)\right] d \zeta^{\prime}\right\} d \tau^{\prime} .
\end{aligned}
$$

This leads to the following formula for the slow rf wave amplitude in the point of the electron beam, $a_{b}(\tau)=a(\tau, \zeta=0)$ : 


$$
a_{b}(\tau)=\exp (-i \alpha \tau) \times \frac{\sqrt{i} G}{2 \sqrt{\pi}} \int_{0}^{\tau} \frac{\hat{j}\left(\tau^{\prime}\right)}{\sqrt{\tau-\tau^{\prime}}} \exp \left(i \alpha \tau^{\prime}\right) d \tau^{\prime},
$$

where $\alpha=\varepsilon^{2} / 2$ describes a small slippage of the radiated $\mathrm{rf}$ wave with respect to the electron bunch. In the simplest approximation of the exact group synchronism $(\alpha=0)$ and the stationary state of the electron bunch $\left(\hat{j}=\right.$ const $\left.=\hat{j}_{0}\right)$, Eq. (32) reduces to the following formula:

$$
a_{b}(\tau)=\frac{\sqrt{i} G}{\sqrt{\pi}} \hat{j}_{0} \times \sqrt{\tau}
$$

Let us compare solutions (30) and (33). If the difference between the wave group velocity and the electron axial velocity is significant, then the radiated wave leaves the electron bunch region fast, so that different "quanta" emitted from the bunch in different moments of time are radiated independently [Fig. 4(a)]. In this case, the rf wave emission has a quasistationary character $\left[a_{b}(t) \approx\right.$ const $]$ described by Eq. (30). In contrast, if the group synchronism takes place $\left(\beta_{\mathrm{gr}}=\beta_{\|}\right)$, then quanta emitted in different moments of time do not leave the bunch region, so that the rf field is accumulated close to the bunch [Fig. 4(b)]; according to Eq. (33), this is described by the growth of the rf wave amplitude, $a_{b}(t) \propto \sqrt{t}$. This is analogous to the superradiation effects, studied both theoretically and experimentally for various types of electron masers [32,33]. Evidently, the use of this superradiant regime should lead to a faster rate of the loss of the transverse electron velocity in the radiation process. Thus, the group synchronism regime is required to provide both conservation of the axial electron velocity (this is required for the "proper" transformation of the "axial velocity cooling" into the "energy
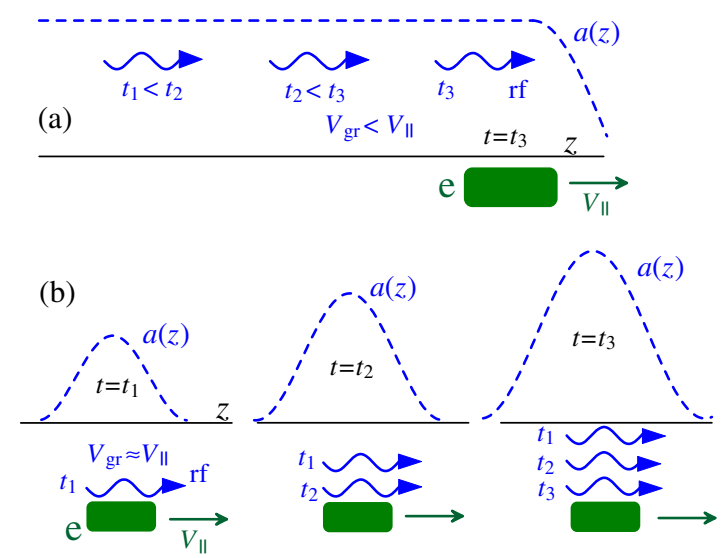

FIG. 4. (a) Portions of the rf wave radiated by the electron bunch in different moments of the time $t_{1}<t_{2}<t_{3}$ and characteristic forms of the wave packet (a) in the case, when the group velocity of the radiated waveguide wave is smaller than the axial electron velocity, and (b) in the case of the group synchronism between electrons and the radiated wave. cooling" in the process of the cyclotron emission) and to increase of the rate of this process.

\section{Estimations and numerical simulations}

In equations of the electron motion (21) and (22), the transition to normalized variables $(\zeta, \tau)$ [see Eq. (25)] can be provided in the following way:

$$
\frac{1}{k_{0}} \frac{d}{d z} \approx \frac{1}{k_{0}}\left(\frac{\partial}{\partial z}+\frac{1}{V_{\|}} \frac{\partial}{\partial t}\right)=\frac{1-\beta_{\|}^{2}}{\beta_{\|}} \frac{\partial}{\partial \tau} \approx \gamma_{0}^{-2} \frac{\partial}{\partial \tau} .
$$

Thus, Eqs. (21) and (22) are transformed as follows:

$$
\frac{\partial p_{\perp}}{\partial \tau} \approx \frac{-1}{2} \times \operatorname{Re} a_{b}(\tau) \exp (i \varphi), \quad \frac{\partial \varphi}{\partial \tau} \approx \frac{\gamma-\gamma_{0}}{\gamma_{0}},
$$

with the initial conditions

$$
p_{\perp}(\tau=0)=p_{\perp 0}=\gamma_{0} \beta_{\perp 0}, \quad \varphi(\tau=0)=\varphi_{0},
$$

where the initial phases are distributed uniformly over interval described by Eq. (23). The electron energy is related with the transverse momentum by the general relativistic relation $\gamma^{2}=1+p_{\perp}^{2}+p_{\|}^{2}$; in the case of $\beta_{\|}=$const, this leads to the following formula:

$$
\gamma=\sqrt{\frac{1+p_{\perp}^{2}}{1-\beta_{\|}^{2}}}
$$

Let us use the "superradiant" approximation (33) for the rf wave amplitude, $a_{b}(\tau)$, as well as the approximation of a small phase size of the electron beam, $\delta \varphi_{0} \rightarrow 0$. Moreover, we assume that $\varphi=$ const $=0$, so that the radiation process does not result in a significant change in the electron energy (and, therefore, in the electron phase); this is true in the case of small enough initial transverse momentum. Then one obtains

$$
p_{\perp}(\tau) \approx \gamma_{0} \beta_{\perp 0}-\frac{\beta_{\perp 0} G}{3 \sqrt{2 \pi}} \tau^{3 / 2}
$$

The normalized time $\tau=\omega_{0}\left(t-V_{\|} z / c^{2}\right)$, which corresponds to a particle with the axial coordinate $z=V_{\|} t$, is related to the length of the path of this particle, $L$, as follows:

$$
\tau \approx \gamma^{-2} \times 2 \pi L / \lambda
$$

Therefore, Eq. (37) leads to the following condition for the length of the radiated section, $L$, which is required to provide the loss of the whole electron transverse momentum, $p_{\perp}(L)=0$ : 


$$
\left(\frac{L}{\lambda}\right)^{3 / 2} \times \frac{L_{e}}{\lambda} \times \frac{I}{I_{A}} \times \gamma_{0}^{-4} \times \frac{8 \pi^{2}}{3 N}=1
$$

As an example, we consider an electron bunch with the total charge $1 \mathrm{nC}$ and the duration $1 \mathrm{ps}$; this corresponds to $I=1 \mathrm{kA}$ and $L_{e}=0.3 \mathrm{~mm}$. We assume also $L_{e}=\lambda / 3$, so that the wavelength of the radiated wave is $\lambda \approx 1 \mathrm{~mm}$. For these parameters, estimation (38) is reduced as follows:

$$
L / \lambda \approx \gamma_{0}^{8 / 3}
$$

In the case of a $5 \mathrm{MeV}$ electron bunch $\left(\gamma_{0} \approx 10\right)$, the radiation section length amounts to $L \sim 500 \lambda=50 \mathrm{~cm}$. An increase in the electron energy up to $\gamma_{0} \approx 50$ results in $L \sim 35000, \lambda=35 \mathrm{~m}$.

Let us notice that parameters mentioned above correspond to a waveguide diameter of $6 \mathrm{~mm}$ at $\gamma_{0} \approx 10$ and to a waveguide diameter of $3 \mathrm{~cm}$ at $\gamma_{0} \approx 50$. This is true in the case of the lowest $\mathrm{TE}_{1,1}$ operating transverse mode; naturally, the system could be more oversized, if the "operating" group synchronism condition $\beta_{\mathrm{gr}}=\beta_{\|}$is organized for a higher transverse mode. In this situation, it is natural (at least, in the simplest model) to neglect the transverse-position-dependent effects (wakefields, inhomogeneity of the transverse structure of the radiated wave), which could be the source of an additional spread in electron energy.

Estimations (38) and (39) are followed from formula (33), which is obtained in the approximation of the constant transverse electron velocity, $\beta_{\perp} \approx \beta_{\perp 0}$. More important, estimation (38) is obtained in the approximation of a small phase size of the electron bunch, $\delta \varphi_{0} \rightarrow 0$. This approximation is true, when the wavelength significantly exceeds the bunch length, $\lambda \gg L_{e}$. However, according to Eq. (38), an increase in the wavelength leads to the increase in the radiation section length. On the other hand, if the bunch phase size is not small, then electrons with different phases have a different rate of the radiation loss of their transverse velocities. At the same time, in order to provide minimization of the spread in energy, one should provide the situation, when all electrons lose their oscillatory velocity almost in the same point.

In order to study this problem, we solve numerically motion equations (34)-(36) together with formula (32) for the rf wave amplitude. We introduce the normalized "transverse kinetic energy" of a particle, $w_{\perp}=$ $\left(\beta_{\perp} / \beta_{\perp 0}\right)^{2}$. Since $\beta_{\|}=$const in the radiation process, and the spread in initial axial velocity has been minimized at the beginning of this process, the spread in relativistic electron energy, $\gamma=1 / \sqrt{1-\beta_{\|}^{2}-\beta_{\perp}^{2}}$, is determined by the spread in $w_{\perp}$ :
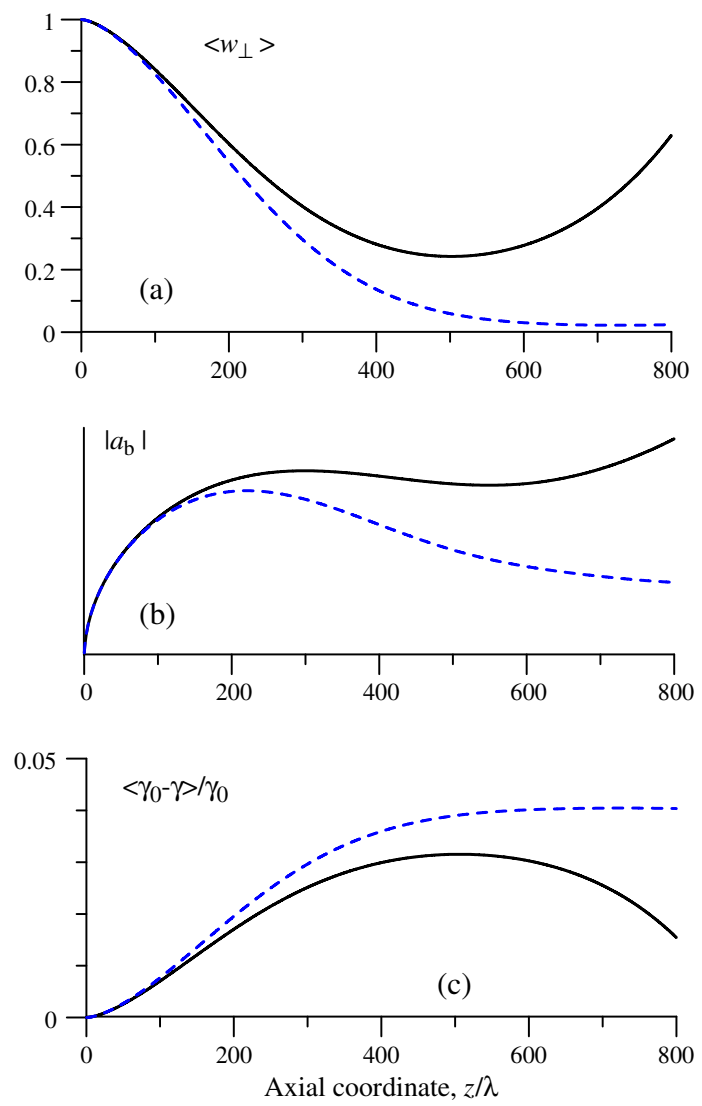

FIG. 5. Results of simulations for the electron energy $\gamma_{0}=10$ : (a) averaged transverse electron energy $\left\langle w_{\perp}\right\rangle$, (b) rf wave amplitude in the point of the electron bunch, and (c) the averaged change in the total electron energy versus the axial coordinate of the bunch. Solid curves illustrate the case of the exact group synchronism $(\alpha=0)$, and dashed curves correspond to the case of the optimal mismatch of the group synchronism $(\alpha=0.072)$.

$$
\frac{\delta \gamma}{\gamma_{0}} \approx \frac{1}{\gamma_{0}} \frac{\partial \gamma}{\partial\left(\beta_{\perp}^{2}\right)} \delta\left(\beta_{\perp}^{2}\right)=\frac{p_{\perp 0}^{2}}{2} \delta w_{\perp} .
$$

Figures 5 and 6 show results of numerical simulations for an electron bunch with $\gamma_{0}=10, I=1 \mathrm{kA}$ and $L_{e}=\lambda / 3$, when the initial phase size of the bunch is quite large $\delta \varphi_{0} / 2 \pi=1 / 3$, and the initial transverse momentum imparted by the kicking undulator is $\gamma_{0} \beta_{\perp 0}=0.3$. Solid curves in Fig. 5 illustrate averaged (over the whole electron ensemble) transverse kinetic energy $\left\langle w_{\perp}\right\rangle$, rf wave amplitude in the point of the electron bunch and the averaged change in gamma factor, $\left\langle\gamma-\gamma_{0}\right\rangle / \gamma_{0}$, versus the axial coordinate of the bunch, $z / \lambda=\gamma^{2} \tau / 2 \pi$, in the case of the exact group synchronism, $\beta_{\mathrm{gr}}=\beta_{\|}$, when the rf wave amplitude is determined by formula (32) at $\alpha=0$. The behavior of transverse kinetic energies $w_{\perp}$ of electrons with different initial phases $0 \leq \varphi_{0} \leq \delta \varphi_{0}$ is illustrated in Fig. 6(a).

In the beginning of the radiation process, the rf wave amplitude increases as predicted by formula (33), 

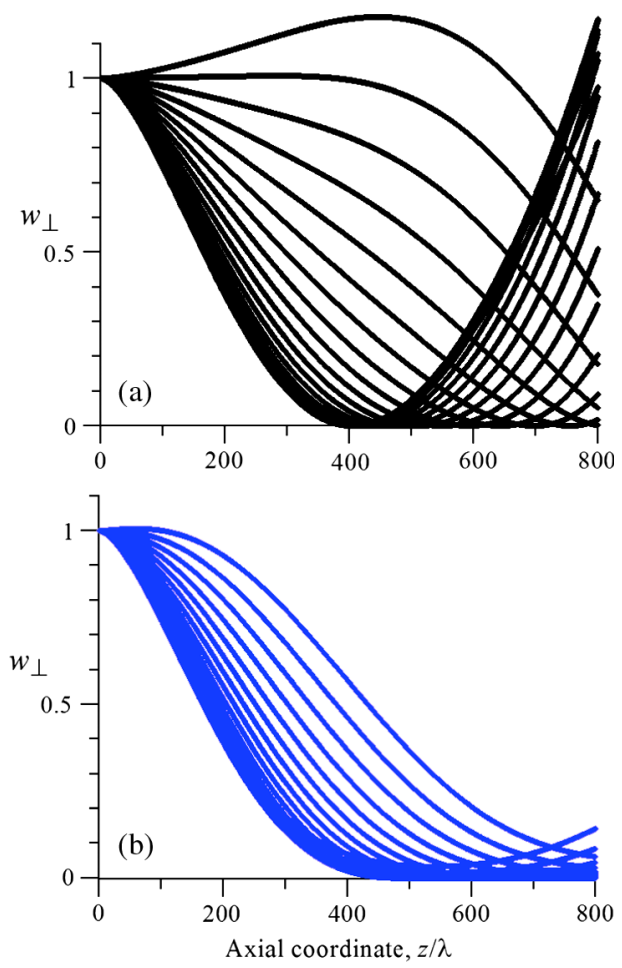

FIG. 6. Results of simulations for the electron energy $\gamma_{0}=10$. Transverse kinetic energies $w_{\perp}$ of electrons with different initial phases versus the axial coordinate of the bunch in the cases of (a) the exact group synchronism $(\alpha=0)$ and (b) the optimal mismatch of the group synchronism $(\alpha=0.072)$.

$a_{b}(z) \propto \sqrt{z}$. However, $a_{b}(z)$ goes to saturation, when the loss in the averaged transverse energy of electrons, $\left\langle w_{\perp}\right\rangle$, becomes significant. Estimations (38) and (39) predict quite exactly the radiation section length corresponding to the maximal loss in the averaged transverse energy, $\left\langle w_{\perp}\right\rangle$ [Fig. 5(a)]. However, this loss is not total; the particles of the bunch lose approximately $70 \%$ of their initial transverse energy. The reason has been mentioned above, namely, this is a different rate of the emission of electrons with different initial phases, $\varphi_{0}$. According to Fig. 6, every particle loses its initial transverse energy, $w_{\perp}=0$, at some point of the axial coordinate. After this point, the cyclotron emission of this particle is changed to the absorption of the energy of the radiated wave propagating together with the particle, such that the transverse energy of this electron increases. Since in the case of the exact group synchronism $\left(\beta_{\mathrm{gr}}=\beta_{\|}\right.$, $\alpha=0$ ) the points of the total loss of the transverse energy, $w_{\perp}=0$, are very different for particles with different initial phases [Fig. 6(a)], the averaged transverse energy of all electrons of the bunch, $\left\langle w_{\perp}\right\rangle$, cannot reach zero.

In order to solve this problem, one should provide the situation, when the group synchronism condition is fulfilled only approximately, $\beta_{\mathrm{gr}} \approx \beta_{\|}$. In this case, the slippage factor $\alpha$ in formula (32) for the rf wave amplitude is not equal to zero. If the bunch phase size is not too large, $\delta \varphi_{0}$, then there is an optimal value of the slippage factor, $\alpha$; at this value, the averaged transverse energy of all electrons of the bunch, $\left\langle w_{\perp}\right\rangle$, becomes very close to zero at some point of the radiation section [Fig. 5(a)]. After this point, the radiation process should be stopped (for instance, by changing the group velocity of the radiated waveguide mode).

In the case of $\gamma_{0}=10$ and $L_{e}=\lambda / 3$, the optimal value of the slippage factor amounts to $\alpha=0.072$. In this case, at the length of the radiation section $z / \lambda=600-800$, transverse energies of all electrons of the bunch becomes close to zero [Fig. 6(b)]. This length is slightly longer than the optimal length in the case of the exact group synchronism $(\alpha=0)$; according to Fig. 5(b), this is due to a smaller amplitude of the rf wave in the case, when there is a slippage between the electron bunch and the radiated $\mathrm{rf}$ signal. As for the loss in the total electron energy caused by the cooling radiation $\left\langle\gamma-\gamma_{0}\right\rangle / \gamma_{0}$, this is as small as several percent [Fig. 5(c)].

Figures 7 and 8 are similar to Figs. 5 and 6, respectively, but they illustrate the cooling process for an electron bunch with a higher energy, $\gamma_{0}=50$. As compared to the case of
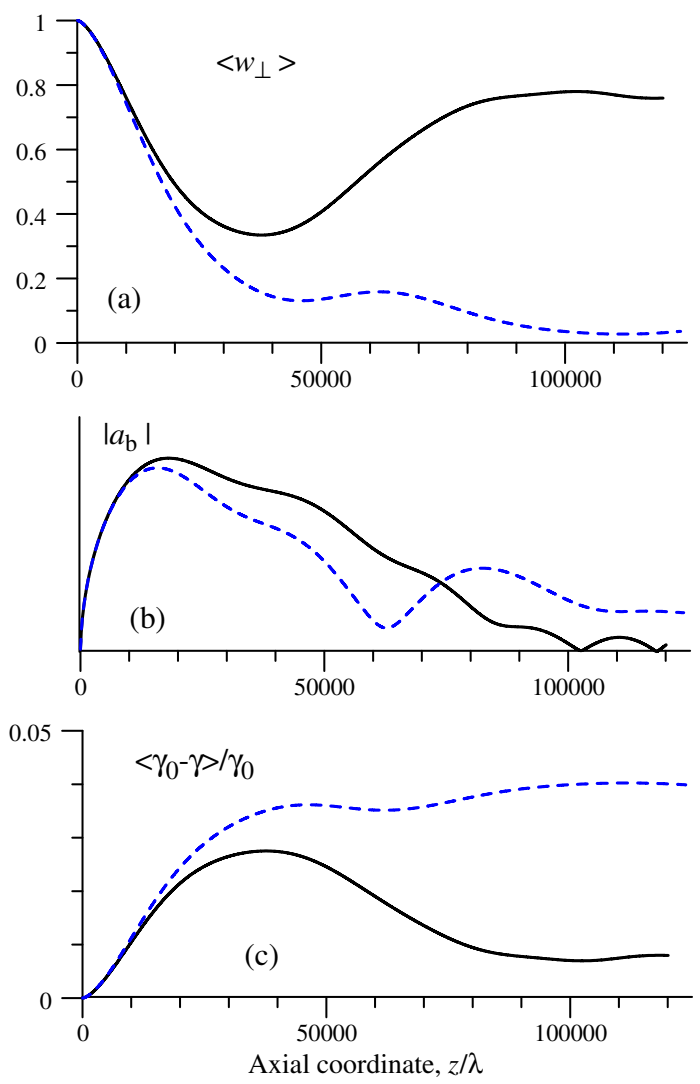

FIG. 7. Results of simulations for the electron energy $\gamma_{0}=50$ : (a) averaged transverse electron energy $\left\langle w_{\perp}\right\rangle$, (b) rf wave amplitude in the point of the electron bunch, and (c) the averaged change in the total electron energy versus the axial coordinate of the bunch. Solid curves illustrate the case of the exact group synchronism $(\alpha=0)$, and dashed curves correspond to the case of the optimal mismatch of the group synchronism $(\alpha=0.014)$. 


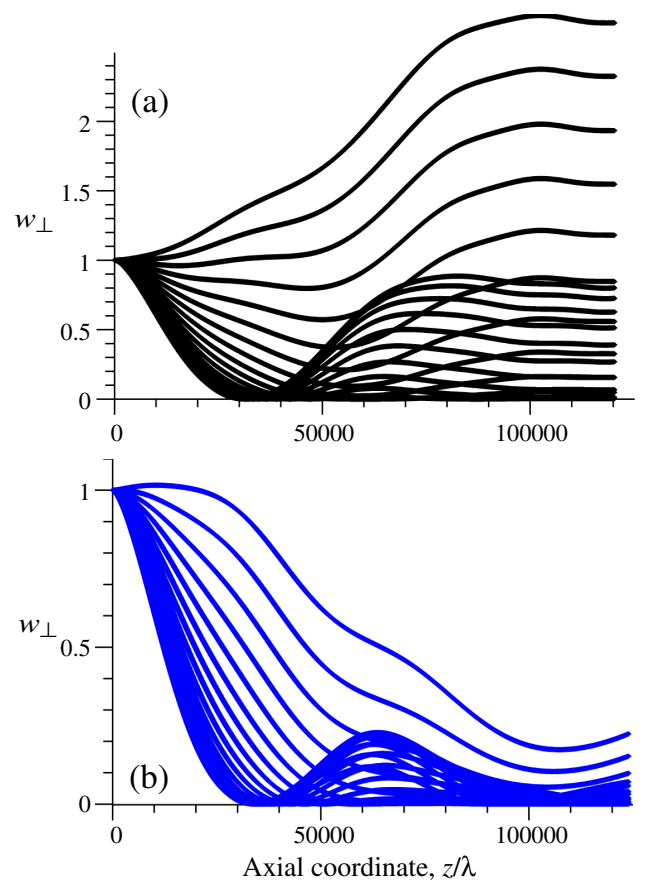

FIG. 8. Results of simulations for the electron energy $\gamma_{0}=50$. Transverse kinetic energies $w_{\perp}$ of electrons with different initial phases versus the axial coordinate of the bunch in the cases of (a) the exact group synchronism $(\alpha=0)$ and (b) the optimal mismatch of the group synchronism $(\alpha=0.014)$.

$\gamma_{0}=10$, the interaction of more heavy electrons with the radiated wave is weaker; correspondingly, the required length of the radiation region is longer. In this situation, the same phase size of the electron bunch $\left(L_{e}=\lambda / 3\right)$ leads to a significantly bigger spread in the electron transverse energy, $w_{\perp}$, arisen in the radiation process. According to Fig. 8(a), in the case of the exact group synchronism $(\alpha=0)$ at $z / \lambda>50000$ the bunch contains both of the emitting electrons, which have lost almost the whole of their initial transverse energies, and absorbing particles with significantly increased transverse energies. In this situation, the radiation provides the loss of only about half of the initial transverse energy of the whole bunch, $\left\langle w_{\perp}\right\rangle$ [Fig. 7(a)]. However, even in this case the proper choice of the slippage factor $(\alpha=0.014)$ provides the situation, when all electrons of the bunch lose their transverse energies almost simultaneously [Fig. 8(b)], and the averaged transverse energy of the bunch becomes close to zero [Fig. 7(a)]. Similar to the case of $\gamma_{0}=10$, the loss in the total electron energy caused by the cooling radiation amounts to several percent [Fig. 7(c)].

Let us discuss these results from the point of view of sensitivity of the proposed system to electron bunch parameters. Although the spread in axial electron velocity is minimized by the kicker (axial cooling undulator), the spread in initial electron energy (in electron cyclotron frequency) exists. The effect of this spread can be easily estimated from Eq. (22). The spread in initial electron energy, $\delta \gamma$, induces the following spread in mismatch:

$$
\delta \Delta \approx \frac{\Omega_{c 0}}{\beta_{\|} \omega_{0}} \frac{\delta \gamma}{\gamma_{0}} \approx\left(\frac{1}{\beta_{\|}}-\beta_{\mathrm{gr}}\right) \frac{\delta \gamma}{\gamma_{0}} \approx \frac{\delta \gamma}{\gamma_{0}^{3}}
$$

The radiation process is sensitive to this spread, if the spread induces a significant spread in electron phases during the motion of the electron bunch through the entire radiation section, $k_{0} L \times \delta \Delta \approx 2 \pi$. This leads to the following simple estimation for the permissible spread in electron energy:

$$
\frac{\delta \gamma}{\gamma_{0}} \approx \gamma_{0}^{2} \frac{\lambda}{L}
$$

In the case of $\gamma_{0}=10$, the radiation section length amounts to $L / \lambda=600-800$ [Fig. 5(a)]; this leads to a reasonable value of the permissible energy spread $\left(\delta \gamma / \gamma_{0} \approx 13-17 \%\right)$. As for the case of $\gamma_{0}=50$, the radiation section length $L / \lambda \approx 10^{5}$ [Fig. 7(a)] corresponds to a permissible energy spread of $\delta \gamma / \gamma_{0} \approx 2.5 \%$. According to estimations (39) and (40), the further increase in the energy should lead to a decrease in the relative permissible energy spread, $\delta \gamma / \gamma_{0} \propto \gamma_{0}^{-2 / 3}$.

An important peculiarity of the electron-wave interaction illustrated by Figs. 5 and 7 is the slowness of this process at the end region, where transverse momenta of all electrons are small and, therefore, their interaction with the rf wave (either the further radiation or the absorption of the wave energy) is weak. Due to this fact, the length of the radiation section is not strongly fixed. In the case of $\gamma_{0}=10$ the length can be varied within a range of $L / \lambda=600-800$ [Fig. 5(a)], such that the possible relative variation is as big as $\delta L / L \approx 30 \%$. This fact makes the cooling process weakly sensitive to nonstability (jitter) of parameters of the electron bunch. Actually, according to Eq. (38), the permissible variations of the electron current in the bunch, $\delta I$, and of the bunch length, $\delta L_{e}$, are related with a variation of the radiation section length as follows:

$$
\frac{\delta I}{I}=\frac{\delta L_{e}}{L_{e}} \approx \frac{3}{2} \frac{\delta L}{L} \approx 45 \%
$$

In the case of $\gamma_{0}=50$ the radiation section length can be varied within a range of $L / \lambda=(1.0-1.2) \times 10^{5}$ [Fig. 7(a)]; thus corresponds to $\delta L / L \approx 18 \%$ and to the following permissible variations of the electron bunch parameters: $\delta I / I=\delta L_{e} / L_{e} \approx 27 \%$. Evidently, the further increase in the electron energy should lead to an increase in the sensitivity of the cooling process to a jitter of bunch parameters. 


\section{CONCLUSION}

In this paper, we propose to use of an undulator with the guiding axial magnetic field as a kicker, which transforms a bunch of rectilinear electrons into a bunch of particles, which move in a uniform axial magnetic field and perform free cyclotron oscillations. At definite conditions, such a kicker can form a bunch with very special properties, namely, the initial (at the input of the kicker) spread in electron energy can be transformed into the spread in oscillatory (transverse) velocity, whereas the spread in the axial velocity is minimized at the output of the kicking system. This effect (axial cooling) can be provided, if the cyclotron electron frequency corresponding to the axial magnetic field exceeds the bounce frequency of electron oscillations in the periodic undulator field. In this situation, electrons with higher input energies obtain bigger undulator oscillatory velocities. In the case of a magnetostatic undulator, the value of the axial magnetic field required to provide this "negative-mass" effect is proportional to the electron gamma factor. The problem of high magnetic fields required to provide axial cooling at high electron energies can be solved by the use of an rf-wave undulator copropagating together with the electron bunch.

The cyclotron emission from the electron bunch leads to losing oscillatory velocity of electron gyrorotation in a magnetic field. However, if the group velocity of the radiated wave (waveguide mode) coincides with the axial electron velocity (the group synchronism condition), then the cyclotron emission does not lead to a change in the axial electron velocity. This effect can be used for transformation of the axial cooling of the electron bunch (minimization of the spread in electron axial velocity) in the undulator section into the "real cooling" (minimization of the spread in electron energy) in the cyclotron radiation section. The group synchronism condition is needed also to provide the maximal rate of the radiation and, therefore, of the cooling process. Another condition of the fast cooling process is the coherent character of the cyclotron radiation; this occurs if the length of the electron bunch is smaller than the wavelength of the radiated wave.

It is important that the cooling of the electron bunch is provided, if all electrons lose their transverse velocities almost in the same point of the cyclotron radiation section. This is provided automatically, if the phase size of the electron bunch is small (the bunch length is negligibly short as compared to the wavelength of the radiated wave). However, shorter wavelengths are more attractive from the point of view of the length of the radiation section. It is shown in this paper that even in the case when the bunch length is comparable with the wavelength, the simultaneous loss of the transverse velocities of all electrons of the bunch can be provided by an optimization of parameters of the microwave system of the radiation section.

The process of cyclotron radiation cooling studied in this paper is strongly dependent on the energy of electrons. First of all, the energy determines the length of the radiation region required to provide the entire loss of the transverse energy of all particles. In the example considered in the paper, this length amounts to several hundreds of wavelengths in the case of a $5 \mathrm{MeV}$ electron bunch, whereas an increase in the electron energy up to $50 \mathrm{MeV}$ results in length as long as $10^{5}$ wavelengths. Naturally, longer lengths of the radiation section cause stronger sensitivity of the cooling process to the spread in electron energy and to a jitter of the electron bunch parameters. Evidently, this is the main factor limiting the usage of the proposed approach for high-energy electron bunches.

In conclusion, we could state that the system described in this paper can be interesting from the point of view of not only electron cooling, but also as a way for creation of a submillimeter-wavelength source of coherent cyclotron radiation from a relativistic electrons bunch. Actually, the proposed way to impart cyclotron rotation to electrons in the axial cooling undulator is attractive, as it provides minimization of the spread in axial electron velocity; the strong sensitivity to this kind of spread is the "Achilles heel" of relativistic electron cyclotron masers with a high Doppler-up-conversion of the electron cyclotron frequency. The use of the coherent spontaneous character of the cyclotron radiation (instead of the stimulated emission) is also attractive, as it provides a high efficiency and a high rate of the extraction of the kinetic electron energy. In addition, the phase of the radiated rf signal is fixed by the short electron bunch; this property can be useful for some applications.

\section{ACKNOWLEDGMENTS}

This work is supported by the Russian Foundation for Fundamental Research, Projects No. 14-08-00803, No. 1402-00691, and No. 15-42-00260.

[1] J. G. Power, in Proceedings of 14th Advanced Accelerator Concepts Workshop (AAC 2010), edited by S. H. Gold and G. S. Nusinovich (AIP, Melville, 2010), p. 163; AIP Conf. Proc. 1299, 20 (2010).

[2] B. Dunham, J. Barley, A. Bartnik, I. Bazarov, L. Cultrera, J. Dobbins, G. Hoffstaetter, B. Johnson, R. Kaplan, S. Karkare et al., Appl. Phys. Lett. 102, 034105 (2013).

[3] F. Stephan, C. H. Boulware, M. Krasilnikov, J. Bahr, G. Asova, A. Donat, U. Gensch, H. J. Grabosch, M. Hanel, L. Hakobyan et al., Phys. Rev. ST Accel. Beams 13, 020704 (2010).

[4] H. P. Freund and T. M. Antonsen, Principles of FreeElectron Lasers (Chapman \& Hall, London, 1996).

[5] Z. Huang and K.-J. Kim, Phys. Rev. ST Accel. Beams 10, 034801 (2007).

[6] C. Bostedt, J. D. Bozek, P. H. Bucksbaum, R. N. Coffee, J. B. Hastings, Z. Huang, R. W. Lee, S. Schorb, J. N. Corlett, P. Denes et al., J. Phys. B 46, 164003 (2013). 
[7] A. M. Sessler, Report No. LBL-38278 UC-427, 1996; Proceedings of the 31st Workshop: Crystalline beams and related issues, Erice, Sicily, Italy, 1995 (World Scientific, Singapore, 1996).

[8] J. L. Hirshfield and G. S. Park, Phys. Rev. Lett. 66, 2312 (1991).

[9] T. Ohgaki and I. Endo, Phys. Rev. ST Accel. Beams 4, 111001 (2001).

[10] D. Mohl and A. M. Sessler, Nucl. Instrum. Methods Phys. Res., Sect. A 532, 1 (2004).

[11] E. G. Bessonov, M. V. Gorbunkov, and A. A. Mikhailichenko, Phys. Rev. ST Accel. Beams 11, 011302 (2008).

[12] H. Deng and C. Feng, Phys. Rev. Lett. 111, 084801 (2013).

[13] I. V. Bandurkin, S. V. Kuzikov, and A. V. Savilov, Appl. Phys. Lett. 105, 073503 (2014).

[14] N. S. Ginzburg and N. Yu. Peskov, Phys. Rev. ST Accel. Beams 16, 090701 (2013).

[15] C. Nielsen and A. Sessler, Rev. Sci. Instrum. 30, 80 (1959).

[16] A. A. Kolomensky and A. N. Lebedev, Atom Energy 7, 549 (1959).

[17] A. V. Gaponov, M. I. Petelin, and V. K. Yulpatov, Radiophys. Quantum Electron. 10, 686 (1967).

[18] V. L. Bratman, Tech. Phys. 46, 2030 (1976).

[19] A. Bondeson and T. M. Antonsen, Jr., Int. J. Electron. 61, 855 (1986)

[20] H. Li and T. M. Antonsen, Jr.,, Phys. Plasmas 1, 714 (1994).

[21] V. L. Bratman and A. V. Savilov, Phys. Plasmas 2, 557 (1995).

[22] A. V. Savilov, Phys. Plasmas 4, 2276 (1997).

[23] V. L. Bratman, O. Dumbrajs, P. Nikkola, and A. V. Savilov, IEEE Trans. Plasma Sci. 28, 633 (2000).
[24] V. L. Bratman, A. E. Fedotov, and A. V. Savilov, Int. J. Infrared Millim. Waves 19, 939 (1998).

[25] H. Jory, R\&D Technology Report No. ECOM01873-F (Varian Associates, Paolo Alto, CA, 1968). See National Technical Information Service Document No. AD675509 (H. Jory, Investigation of Electronic Interaction with Optical Resonators for Microwave Generation and Amplification). Copies may be ordered from the National Technical Information Service, Springfield, VA 22161.

[26] V. L. Bratman, A. E. Fedotov, Y. K. Kalynov, V. N. Manuilov, M. M. Ofitserov, S. V. Samsonov, and A. V. Savilov, IEEE Trans. Plasma Sci. 27, 456 (1999).

[27] M. I. Petelin, Radiophys. Quantum Electron. 17, 686 (1974).

[28] V. L. Bratman, N. S. Ginzburg, and M. I. Petelin, Opt. Commun. 30, 409 (1979).

[29] V. L. Bratman, N.S. Ginzburg, G. S. Nusinovich, M. I. Petelin, and P. S. Strelkov, Int. J. Electron. 51, 541 (1981).

[30] V. L. Bratman, G. G. Denisov, N. S. Ginzburg, and M. I. Petelin, IEEE J. Quantum Electron. 19, 282 (1983).

[31] A. V. Savilov, Phys. Rev. E 64, 066501 (2001).

[32] N. S. Ginzburg, I. V. Zotova, and A. S. Sergeev, JETP Lett. 60, 513 (1994).

[33] N. S. Ginzburg, A. S. Sergeev, I. V. Konoplev, I. V. Zotova, A. D. R. Phelps, A. W. Cross, S. Cook, P. Aitken, V. G. Shpak, M. I. Yalandin, and S. A Shunailov, Phys. Rev. Lett. 78, 2365 (1997).

[34] R. D. Richtmyer, Principles of Advanced Mathematical Physics (Springer-Verlag, Berlin, 1978), Vol. 1. 